\title{
Aspectos de Qualidade em Jogos Sérios Digitais
}

\author{
Mateus A. Sousa e Silva - UnB - mateusaugusto-2009@hotmail.com, \\ https://orcid.org/0000-0001-8815-5506 \\ André Barros de Sales - UnB - andrebdes@unb.br, \\ https://orcid.org/0000-0002-1728-6063 \\ Fabiana Freitas Mendes - UnB - fabianamendes@unb.br, \\ https://orcid.org/0000-0002-1724-2044
}

Resumo: Os jogos vêm ganhando cada vez mais espaço como uma ferramenta pedagógica em diversas áreas de conhecimento. Nesse contexto, é importante ressaltar que é necessário um processo robusto de desenvolvimento centrado no jogador, cujas características e experiências almejadas pelo usuário tenham o maior foco. Para isso, este artigo objetiva analisar os aspectos de qualidade em jogos sérios digitais para apoiar o processo de ensino e aprendizagem de estudantes da área de Ciência da Computação. Os aspectos são os requisitos de qualidade do jogo e a experiência do usuário (jogador). Foi realizado um survey a fim de entender a relevância destes aspectos. Houveram 166 participantes e a partir das respostas deles, foi elaborado um ranking dos requisitos de qualidade e experiência do jogador mais importantes para a amostra. No aspecto de requisitos de qualidade os dois principais foram e jogo ter um design atraente e oferecer feedback enquanto para a experiência do jogador foram o jogo gerar o sentimento de satisfação em jogar e aprender e produzir o sentimento de confiança que o próprio jogador vai aprender o conteúdo proposto pelo jogo.

Palavras-chave: Jogos Sérios, Jogos para Aprendizagem, Requisitos Qualidade, Experiência do Usuário.

\section{Quality Aspects in Serious Digital Games}

Abstract: Games have been gaining more and more space as a pedagogical tool in several areas of knowledge. In this context, it is important to emphasize that a robust development process centered on the player is needed, whose characteristics and experiences desired by the user have the greatest focus. For that, this article aims to analyze the quality aspects in serious digital games to support the teaching and learning process of students in the field of Computer Science. Aspects are game quality requirements and user (player) experience. A survey was carried out in order to understand the relevance of these aspects. There were 166 participants and based on their responses, a ranking of the most important quality requirements and player experience for the sample was drawn up. In terms of quality requirements, the two main ones were that the game has an attractive design and offers feedback, while for the player experience it was the game generating the feeling of satisfaction in playing and learning and producing the feeling of confidence that the player will learn the content proposed by the game.

Keywords: Serious Games, Games for Learning, Quality Requirements, User Experience.

\section{Introdução}

A academia tem investido em novas abordagens e tecnologias como recursos auxiliares no processo de aprendizagem, auxiliando no desenvolvimento de atividades pedagógicas inovadoras e colaborativas em diversas áreas (Battistella, 2016; Brito et al., 
2016; Sales; Serrano e Serrano, 2020). Os Jogos Sérios fazem parte dessas abordagens, que vem se tornando cada vez mais populares na educação em computação, pois podem aumentar a eficácia e o engajamento da aprendizagem (Battistella, 2016; Brito et al., 2016; Sales; Clímaco e Sales, 2016; Queiroz et al., 2017). Esse tipo de jogo pode ser aplicado em diversas áreas, tais como saúde, publicidade, política e educação. Jogos Sérios também incluem Jogos para Aprendizagem (Becker, 2021).

Com o avanço tecnológico do século XXI, as pessoas estão cada vez mais tendo acesso e utilizando os jogos digitais, e hoje em dia, se tornou um mercado enorme. Segundo o SuperData (2021), no relatório anual de 2020, a indústria de jogos gerou 126,6 bilhões de dólares, sendo 73,8 bilhões de dólares desse lucro gerado por jogos digitais para dispositivos móveis.

No desenvolvimento de um jogo, partindo da concepção de uma ideia até um resultado satisfatório para os usuários, é necessário um processo sólido de design de jogos (Fullerton, 2008, p. 10-11). De acordo com Fullerton (2008) o ponto chave para tal processo é definir para o jogo, metas de experiência para os jogadores-alvo. Para isto é preciso ter o entendimento sobre público ao qual o jogo será destinado faz-se necessário elicitar as características do jogador, tra çando assim um perfil de usuário ou elaborando personas (Barbosa et al., 2021, p. 151-157).

Esta é a visão compartilhada pela área de Interação Humano-Computador (IHC), na qual o desenvolvimento de sistemas interativos é centrado no usuário, prezando pela qualidade de uso destes sistemas e o seu impacto na vida dos usuários (Barbosa et al., 2021, p. 8-9). A busca por esta qualidade envolve estudos sobre a interação entre seres humanos, os sistemas computacionais e elementos relacionados. $\mathrm{O}$ conhecimento sobre esse assunto permite aos profissionais de design e de desenvolvimento dessas tecnologias, a possibilidade de construí-las, melhorá-las e inseri-las na vida das pessoas sempre buscando uma boa experiência dos usuários (Sales; Costa e Alcântara, 2017; Barbosa et al., 2021, p. 13-14).

Portanto, o objetivo deste trabalho é analisar aspectos de qualidade em jogos sérios digitais que auxiliem o processo de ensino e aprendizagem de estudantes de graduação e pós-graduação na área de Ciência da Computação. Os aspectos de qualidades considerados são: requisitos de qualidade do jogo e experiência do usuário.

Este artigo se estrutura em cinco seções, sendo esta a Seção 1, introduzindo a pesquisa, seguido da Seção 2, na qual são apresentados trabalhos relacionados ao tema. Na Seção 3 é apresentada a metodologia adotada e em seguida a Seção 4, que apresenta e discute os resultados obtidos. Por fim, a Seção 5 apresenta as considerações finais.

\section{Trabalhos Relacionados}

Este artigo é um dos resultados do projeto de pesquisa Recursos Digitais Didáticos para Interação Humano-Computador. Dentre os resultados deste projeto de pesquisa encontram-se (Sales e Antunes, 2021; Sales e Silva, 2020). Sales e Silva (2020) realizaram uma Revisão Sistemática da Literatura (RSL), que identificou Jogos para Aprendizagem na área de IHC. Da análise destes jogos obteve-se os requisitos de qualidade: "facilidade na interação do usuário com o jogo", "execução das atividades do jogo em tempo adequado (período de uma aula de curso de graduação)", "abordagem dos conteúdos de forma a facilitar a aprendizagem", "feedbacks", "narrativa", "pontos e recompensas"e "ranking dos jogadores". Já em relação a experiência dos usuários ao usarem esses jogos, obteve-se os aspectos desejáveis "criatividade", "motivação", "atratividade", "satisfação", "diversão" e "desafio". E os aspectos indesejáveis foram 
"beleza forçada", "apreensão" e "desapontamento".

Durante o processo de estudo do tema, além da RSL, foi encontrado o trabalho de Petri e Wangenheim (2019). Este apresenta o MEEGA+, um instrumento de medição e avaliação da qualidade de jogos educacionais. O instrumento foi construído com base nos resultados de revisões de literatura e de uma análise estatística exploratória de estudos de caso. O MEEGA+ é composto por dois fatores de qualidade (usabilidade e experiência do jogador), cada qual com suas dimensões (Petri e Wangenheim, 2019).

O fator de usabilidade é composto pelas seguintes dimensões: estética, capacidade de aprendizagem, operabilidade e acessibilidade. Já o fator da experiência do jogador é composto pelas dimensões: atenção focada, diversão, desafio, interação social, confiança, relevância, satisfação e aprendizagem percebida (Petri e Wangenheim, 2019). Estes dois trabalhos foram utilizados como base para o desenvolvimento desta pesquisa. Este desenvolvimento é apresentado na seção a seguir, a qual explica suas etapas metodológicas.

\section{Metodologia de Pesquisa}

De acordo com Gerhardt e Silveira (2009, p. 39), a pesquisa aqui relatada pode ser classificada, quanto a sua abordagem, como do tipo quali-quantitativo, quanto ao objetivo, este estudo tem cunho descritivo e sobre o procedimento, e do tipo pesquisa com survey, utilizando de um questionário auto-aplicável. De acordo com Kasunic (2005) um survey possui sete etapas, conforme ilustra a Figura 1.

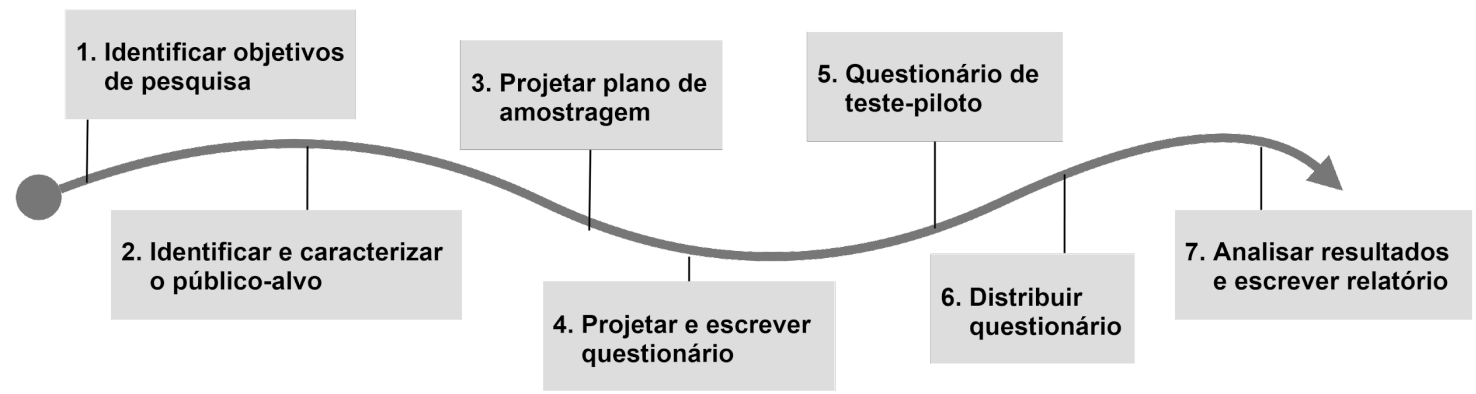

Figura 1. Visão Geral do Processo de Pesquisa de um Survey - Traduzido de Kasunic (2005)

O processo se inicia com a identificação do objetivo de pesquisa como é apresentado na primeira etapa da Figura 1. Foi definido o objetivo do survey como "identificar a relevância dos aspectos de qualidade em jogos sérios no processo de ensino e aprendizagem". Seguindo na Figura 1, na segunda etapa tem-se a identificação e caracterização do público-alvo, que neste trabalho são uma população de alunos de graduação e pós-graduação de cursos da área de Ciência da Computação.

A etapa 3 da Figura 1 contempla a realização do plano de amostragem, definido para ser executado dentro de instituições federais de ensino de graduação em pós-graduação, coletando os dados via questionário virtual, entre os dias 06/10/2020 e 27/10/2020. O questionário elaborado na etapa 4, conforme se segue na Figura 2, teve por base os trabalhos de (Petri e Wangenheim, 2019) e de Sales e Silva (2020).

O questionário passou por algumas rodadas de teste, correções e melhorias, como prescreve a quinta etapa (Figura 1) e além de detectar quaisquer outros erros e contabilizar o tempo médio para se realizar o questionário, o teste também verificou se as questões estavam fáceis de serem entendidas e se o layout e fluxo estavam adequados. O questionário contou com 11 questões, sendo as primeiras quatro referentes a informações demográficas, e as demais são apresentadas na Tabela 1. 
Na sequência, na etapa 6 (Figura 1), o questionário foi distribuído via mensagem eletrônica e redes sociais para a comunidade discente da Universidade Católica de Salvador (UCSAL), Universidade de Brasília (UnB), Universidade Federal de Uberlândia (UFU), Universidade Federal do Amazonas (UFAM), Universidade Federal do Mato Grosso (UFMT), Universidade Federal do Mato Grosso do Sul (UFMS) e Universidade Tecnológica Federal do Paraná (UTFPR).

Tabela 1. Questionário de Pesquisa

Q1 - Você já usou jogos para aprender algum conteúdo? ${ }^{1}$

Questão fechada, com as opções: Já usei, mas não jogo mais, Eu uso, Não uso, mas tenho interesse em jogar, não uso e não tenho interesse em jogar.

Q1.1 - Quais motivos levaram você a usar esse tipo de jogo? ${ }^{2}$

Questão mista, de múltiplas escolhas, com as opções: Aprender um conteúdo novo, Revisar um conteúdo, Avaliar meu conhecimento sobre um conteúdo e Outro.

Q1.2 - Por que você não tem interesse nesse tipo de jogo?

Questão mista, de múltiplas escolhas, com as opções: Não conheço nenhum, Não gosto de jogos, Não tenho dispositivos eletrônicos para jogar, Não considero que jogos podem me ajudar no processo de aprendizagem e Outro.

Q2 - Qual a relevância dos elementos abaixo, para esse tipo de jogo ? ${ }^{3}$

Questão fechada, de múltiplas escolhas, com as opções: Não é importante, Pouco importante, Indiferente, Importante e Muito importante.

Q3 - Qual a importância dos elementos abaixo para uma boa experiência com esse tipo de jogo ? $^{4}$

Questão fechada, de múltiplas escolhas, com as opções: Não é importante, Pouco

importante, Indiferente, Importante e Muito importante.

$\mathrm{Na}$ última etapa, conforme mostra a Figura 1, os dados coletados foram armazenados em uma planilha eletrônica e posteriormente analisados. O procedimento, o questionário, os dados e a análise completa e com mais detalhes, encontra-se num pacote de pesquisa no repositório do Github ${ }^{5}$.

Foram coletadas 184 respostas, porém com inconsistências em algumas respostas: oito pessoas responderam o questionário mais de uma vez, e dez pessoas não cursaram graduação na área de computação. Essas dezoito respostas foram removidas restando, portanto, um total de 166 respostas válidas. Em relação às características demográficas, a idade dos respondentes varia entre 18 e 53 anos, sendo que $75 \%$ deles possuem até 23 anos de idade. Em relação ao sexo, 128 respondentes $(77 \%)$ são do sexo masculino, $36(22 \%)$ do sexo feminino e dois dos respondentes preferiram não responder a essa pergunta. Dentre as instituições de ensino que participaram da pesquisa, foram obtidas 126 respostas de alunos ou ex-alunos da UnB, 24 da UFMS, 10 da UFAM, 4 da UFMT, 1 resposta da UCSAL e outra da UTFPR.

\footnotetext{
${ }^{1}$ Ao optar por "Já usei, mas não jogo mais", ou "Eu uso", ou "Não uso, mas tenho interesse em jogar" o respondente é direcionado a questão Q1.1, ma se optar por "Não uso e não tenho interesse em jogar" ele é direcionado para a questão Q1.2

${ }^{2}$ A questão mista contém itens com respostas fechadas e um item, "Outro", como resposta aberta.

${ }^{3}$ Os elementos da questão Q2 estão na Tabela 2, com os indicadores de prioridade de 01 a 12.

${ }^{4}$ Os elementos da questão Q3 estão na Tabela 3, com os indicadores de prioridade de 01 ao 08.

${ }^{5} \mathrm{https}$ :/github.com/RecursosDigitaisdeEnsinoAprendizagemIHC/research-pack-hci-games
} 
Em relação à interação dos alunos com jogos para aprendizagem, o resultado identificou que 61\% (102 respondentes) já usaram algum jogo, mas não usavam mais; que $31 \%$ (51 respondentes) ainda usavam jogos para aprender algum conteúdo; e que $8 \%$ (13 respondentes) nunca haviam usado esse tipo de jogo. Destes que nunca haviam usado jogos para aprendizagem, 11 tinham o interesse de jogar, 1 considerava que jogos não auxiliam no processo de aprendizagem e o outro não gostava de jogos.

Das opções das questões de múltiplas escolhas (Q1.1 e Q1.2, Tabela 1) sobre as motivações no uso de jogos para aprendizagem observa-se: 57\% (95 respondentes) possuem a intenção em aprender um novo conteúdo; 56\% (93 respondentes) possuem interesse de avaliar o nível de conhecimento; e 38\% (64 respondentes) possuem o objetivo de revisar um conteúdo. Além disso, 6\% (10 respondentes) apontaram outros motivos para se usar esses jogos, uns relacionaram ao sentimento de diversão, curiosidade e a busca por lazer e entretenimento, já outros relacionaram o motivo do uso do jogo com atividade necessária para um projeto ou disciplina acadêmica.

Os motivos de uso dos jogos ainda podem ser relacionados à interação que os usuários tiveram com eles. As interações são: aqueles que já jogaram, mas não usam mais jogos para aprender algum conteúdo; aqueles que usam esse tipo de jogo; e os que nunca jogaram, mas tem interesse em usá-los. A Figura 2 mostra essa relação entre o tipo de interação que os usuários tiveram com jogos para aprendizagem e a sua motivação de uso. Cada motivação no uso dos jogos se encontra subdividida pelo tipo de interação que os usuários tiveram com os jogos.

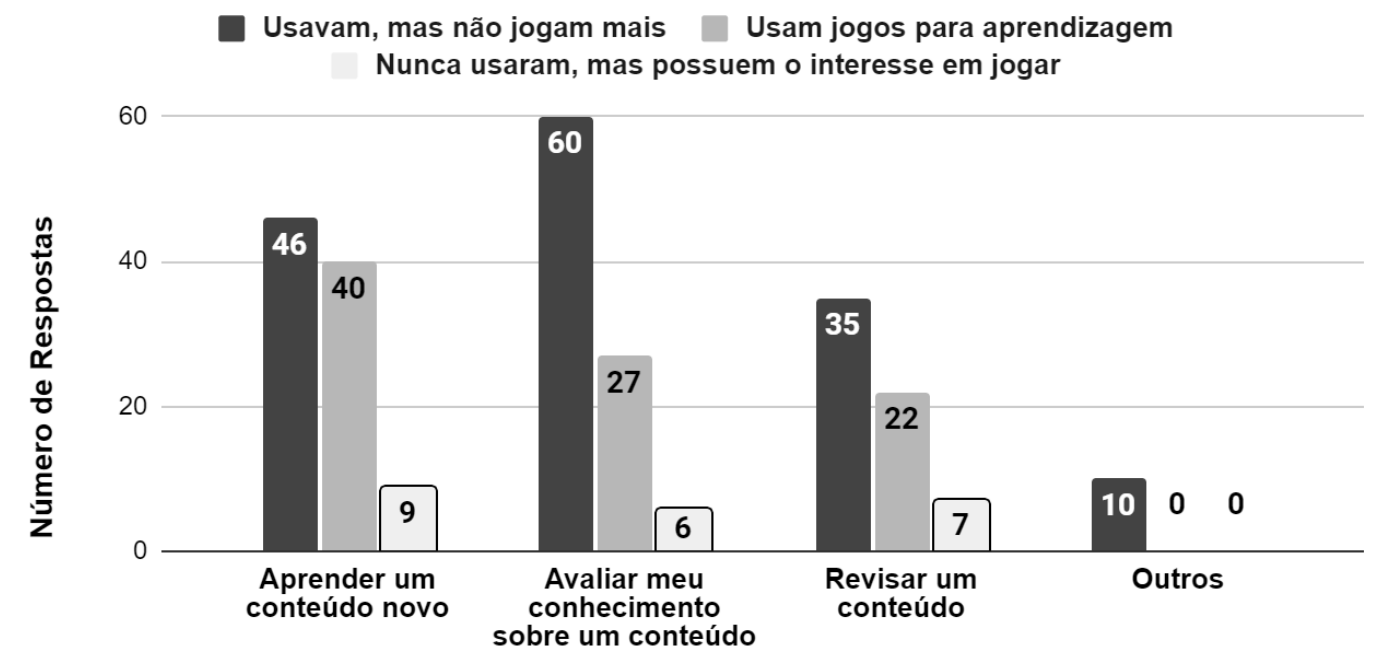

Figura 2. Relação da interação dos usuários com os jogos e sua motivação de uso.

Dos 57\% (95 respondentes) que possuem a motivação de aprender um novo conteúdo, 46 respondentes usavam, mas não jogam mais, 40 usam esses jogos e 9 nunca usaram, mas possuem o interesse em jogar. Observou-se que a maioria dos que usavam, mas não jogam mais (60 respondentes), preferiam jogos para avaliar o conhecimento sobre algum conteúdo. Já a maior parte dos jogadores que ainda jogam (40 respondentes) e daqueles que nunca usaram, mas possuem interesse em jogar ( 9 respondentes), gostariam de aprender um conteúdo novo através de jogos.

Por fim, na etapa de análise, foi definida uma pontuação para cada um dos requisitos de qualidade e de experiência do jogador, a fim de identificar sua relevância para alunos de graduação e pós-graduação na área de Ciência da Computação. A definição dessa pontuação consiste no somatório do grau de relevância dado por cada 
respondente a cada um destes aspectos de qualidade. Os graus de relevância foram convertidos em valores numéricos, sendo eles Não é importante, Pouco importante, Indiferente, Importante e Muito importante por 0, 1, 2, 3 e 4, respectivamente.

\section{Apresentação e Discussão dos Resultados}

Os aspectos de qualidade em jogos para aprendizagem que foram analisados estão agrupados em requisitos de qualidade e experiência do jogador. Foi definida uma pontuação para cada um dos aspectos, variando de 0 a 664 pontos. Esses limites remetem ao caso de se todos os respondentes dessem o grau de relevância mínimo (0) ou todos dessem o grau de relevância máximo (4) para cada um dos aspectos de qualidade. Na Tabela 2 encontram-se ranqueados os requisitos de qualidade ordenados pela relevância definida pelos respondentes. Observa-se que dentre eles os mais apreciados pelos jogadores são: o jogo ter um design atraente, oferecer feedback e ter regras fáceis e claras de se entender. Já os menos relevantes pelos jogadores são: oferecer pontos e recompensas, apresentar ranking dos jogadores e possuir uma narrativa ou história.

Tabela 2. Classificação dos Requisitos de Qualidade Apreciados pelos Usuários

\begin{tabular}{|c|l|c|l|}
\hline Classificação & Requisito de Qualidade & Pontuação & Fonte \\
\hline 01 & Design do jogo atraente & 564 & PW, SS \\
\hline 02 & Oferece feedback ao jogador & 553 & SS \\
\hline 03 & Regras fáceis e claras de se entender & 544 & PW \\
\hline 04 & Facilidade em aprender a jogar & 542 & PW, SS \\
\hline 05 & Padrão de design consistente & 538 & PW \\
\hline 06 & Fontes usadas serem fáceis de ler & 522 & PW \\
\hline 07 & Facilidade em jogar & 513 & PW \\
\hline 08 & Acessibilidade & 506 & PW \\
\hline 09 & Uso adequado das cores & 503 & PW \\
\hline 10 & Oferece pontos e recompensas & 480 & SS \\
\hline 11 & Apresenta ranking dos jogadores & 404 & SS \\
\hline 12 & Possui uma narrativa ou história & 340 & SS \\
\hline
\end{tabular}

Legenda: SS - Sales e Silva (2020); PW - Petri e Wangenheim (2019)

Os requisitos de qualidade são conhecidos também como requisitos não-funcionais ou ainda requisitos de restrição. Segundo SWEBOK (Bourque et al. 2014), estes requisitos atuam para restringir uma solução de software, além de influenciarem na experiência do usuário. Pode ser citado a usabilidade, que segundo a NBR-ISO/IEC 9126-1 (2003) é um requisito de qualidade de software.

Nielsen (1993) define critério de usabilidade como um conjunto de fatores que qualificam quão bem uma pessoa pode interagir com um sistema interativo, esses critérios são: facilidade de aprendizado, facilidade de recordação, eficiência, segurança no uso, satisfação do usuário. Preece, Rogers e Sharp (2005) definem esses critérios de usabilidade como metas, que buscam "assegurar que produtos interativos sejam fáceis de aprender a usar, eficazes e agradáveis, na perspectiva do usuário".

Alguns dos requisitos apresentados na Tabela 2 podem se relacionar aos critérios de usabilidade apresentados por Nielsen (1993). O requisito de qualidade "Design do V.19 $\mathrm{N}^{\circ} 1$, julho, 2021 RENOTE 
jogo ser atraente" e do jogo ter um "Padrão de design consistente" apresentados, na Tabela 2, encontram-se classificados na primeira e quinta posição respectivamente. Estes podem ser relacionados ao critério "satisfação do usuário" definido por Nielsen (1993). "Satisfação do usuário" é definida como "o fator de usabilidade relacionado com uma avaliação subjetiva que expressa o efeito do uso do sistema sobre as emoções e os sentimentos do usuário" (Barbosa et al., 2021, p. 37). Em relação a um jogo e no que foi levantado em questão na pesquisa, essa satisfação é refletida na avaliação do usuário quanto a identidade visual, o tema e combinação de elementos gráficos do jogo.

O requisito "Regras fáceis e claras de se entender", classificado na terceira posição, pode ser relacionado com o critério "facilidade de recordação" definido por Nielsen (1993). "Facilidade em recordação" refere-se ao "esforço cognitivo do usuário necessário para lembrar como interagir com a interface do sistema interativo, conforme aprendido anteriormente" (Barbosa et al., 2021, p. 36), que no caso de um jogo se reflete no esforço para se lembrar de suas regras.

O requisitos "Facilidade em aprender a jogar" pode ser relacionado com o princípio de usabilidade "facilidade de aprendizagem" definido por Nielsen (1993). "Facilidade de aprendizado" remete ao "tempo e esforço necessários para que o usuário aprenda a utilizar o sistema com determinado nível de competência e desempenho" (Barbosa et al., 2021, p. 36), que em um jogo seria a facilidade de se aprender a jogá-lo.

Os requisitos "Design do jogo ser atraente" e "Padrão de design consistente" acabam impactando no aspecto de "Acessibilidade", classificada na oitava posição Tabela 2, que por sua vez está ligado aos aspectos de "Fontes usadas serem fáceis de ler" e "Uso adequado das cores", na sexta e nona posição respectivamente. Estes aspectos citados podem ser relacionados ao critério de acessibilidade em si, definido como "a capacidade do usuário acessar o sistema para interagir com ele, sem que a interface imponha obstáculos" (Barbosa et al., 2021, p. 48). Num jogo isto seria referente desde casos mais gerais, como as cores e as fontes de texto não dificultarem a identificação de um elemento do jogo, como também poderia ser relacionado a casos mais específicos, no qual o jogo teria elementos para incluir algum usuário com limitações motoras, cognitivas ou nos sentidos.

O requisito classificado na segunda posição "Oferece feedback ao jogador" está relacionado a "visibilidade do estado do sistema", no qual feedback de um sistema é definido como "um retorno de informações a respeito de que ação foi feita e do que foi realizado, permitindo a pessoa continuar a atividade" (Preece; Rogers e Sharp, 2005). Em um jogo isso pode ser refletido por exemplo, numa mensagem de Game Over, uma mensagem de que foi realizada uma ação errada, ou que um inimigo se aproxima.

O requisito "Regras fáceis e claras de se entender" pode influenciar na "Facilidade em jogar", classificado na sétima posição (Tabela 2), e ambos podem se relacionar à operacionalidade. A operacionalidade é definida como "capacidade do produto de software de possibilitar ao usuário operá-lo e controlá-lo" (NBR-ISO/IEC 9126-1, 2003). A operacionalidade, por sua vez, tem uma relação com a eficiência (Nielsen, 1993; NBR-ISO/IEC 9126-1, 2003; Preece; Rogers e Sharp, 2005).

A eficiência é o "tempo necessário para conclusão de uma atividade com apoio computacional. Esse tempo é determinado pela maneira como o usuário interage com a interface do sistema" (Barbosa et al., 2021, p. 36). O requisito "Facilidade em Jogar"se relaciona com a eficiência, em um jogo pode ser observado na complexidade de se executar comandos, apertar botões e realizar atividades.

Por fim, os requisitos "Oferece pontos e recompensas", "Apresenta ranking dos 
jogadores" e se "Possui uma narrativa ou história", respectivamente definidos na décima, décima primeira e décima segunda classificação, são bem específicos de jogos. Eles estão relacionados a artifícios para constituição da relação entre o objetivo do jogo, as regras, os feedbacks oferecidos e a participação do jogador (Vianna et al., 2013).

Na Tabela 3 encontram-se ranqueadas as experiências ordenadas pela relevância definida pelos respondentes. Destas experiências as mais buscadas são o sentimento de satisfação em jogar e aprender, ter confiança que o próprio jogador vai aprender o conteúdo proposto pelo jogo e também que o jogador vai perceber a relevância do conteúdo ensinado. As experiências menos relevantes são: ter a atenção focada durante o jogo, o jogo como o principal meio de aprendizagem e interagir com outros jogadores.

Tabela 3. Classificação da Experiência Apreciada pelos Usuários

\begin{tabular}{|c|l|c|l|}
\hline Classificação & Experiência apreciada pelos jogadores & Pontuação & Fonte \\
\hline 1 & Sentir satisfação em jogar e aprender & 600 & PW,SS \\
\hline 2 & Ter confiança de que aprenderei o conteúdo & 558 & PW \\
\hline 3 & Perceber a relevância do conteúdo ensinado & 554 & PW \\
\hline 4 & Me divertir & 539 & PW,SS \\
\hline 5 & Me sentir desafiado & 536 & PW,SS \\
\hline 6 & Ter a atenção focada durante o jogo & 528 & PW \\
\hline 7 & O jogo como principal meio de aprendizagem & 361 & PW \\
\hline 8 & Interagir com outros jogadores & 360 & PW,SS \\
\hline
\end{tabular}

Legenda: SS - Sales e Silva (2020); PW - Petri e Wangenheim (2019)

Segundo Wiemeyer et al. (2016), conforme citado por Barbosa et al. (2021, p. 47), a experiência do jogador é descrita como a qualidade das interações jogador-jogo, e é tipicamente investigada durante e após a interação com os jogos. Preece, Rogers e Sharp (2005) definem algumas metas de experiência de usuário, e estas podem ser aspectos que o usuário considera desejáveis ou indesejáveis.

Dentre estes aspectos que Preece, Rogers e Sharp (2005) pontuam, destacam-se os aspectos desejáveis e indesejáveis que podem ser relacionados aos aspectos de experiência do jogador. Identificados nesta pesquisa, foram apenas os aspectos desejáveis, tais como: Satisfatório, Motivador, Recompensador, Divertido, Desafiador, Interessante, Atraente e Melhora a sociabilidade.

\section{Considerações Finais}

Neste trabalho foi executado um survey, cujo foco foi evidenciar os aspectos de qualidade de jogos sérios digitais para aprendizagem, que contou com a participação de 166 estudantes de graduação em pós-graduação na área de Ciência da Computação. Como resultado, obteve-se um ranking dos requisitos de qualidade mais apreciados pelos jogadores e outro em relação às experiências mais relevantes.

Foram classificados por ordem de relevância, doze requisitos de qualidade e oito experiências apreciadas pelos jogadores. Dentre os requisitos de qualidade, o mais relevante é o design atraente e o menos relevante é a narrativa ou história do jogo. Já em relação às experiências, o que se destaca é o sentimento de satisfação em jogar e aprender e o que recebeu menor destaque foi a interação entre jogadores.

Foi observado que estes requisitos de qualidade refletem critérios, metas de 
usabilidade e até mesmo princípios de design encontrados na literatura (Nielsen, 1993; NBR-ISO/IEC 9126-1, 2003; Preece; Rogers e Sharp, 2005; Barbosa et al., 2021). Além da relação entre as experiências de jogador identificadas na pesquisa e os aspectos de experiência desejáveis apresentados por Preece, Rogers e Sharp (2005).

Estes resultados fornecem aos designers e desenvolvedores de jogos uma lista priorizada das experiências esperadas pelos jogadores e outra dos requisitos de qualidade por ordem de sua relevância, além do entendimento sobre o perfil do público-alvo, para o qual pode-se desenvolver jogos para aprendizagem. Estes insumos são norteadores para a elaboração de um jogo, como é o caso do Playcentric Design Process, um processo de desenvolvimento de jogos, que tem como ponto chave de sua metodologia as metas de experiência do jogador (Fullerton, 2008).

Além do uso na indústria de jogos, este trabalho pode contribuir em futuras pesquisas acadêmicas, avançando no estudo até então realizado. Como por exemplo, sobre a generalização dos resultados é importante ressaltar, que os resultados desta pesquisa dizem respeito, apenas a amostra analisada de alunos de graduação e pós-graduação em cursos de Ciência da Computação, logo, caso o mesmo questionário seja aplicado a uma amostra com características diferentes, tais como, outras áreas de conhecimento ou diferentes graus de escolaridade, será possíivel observar resultados diferentes aos aqui apresentados.

Como trabalhos futuros, esse mesmo estudo poderia ser feito objetivando populações de outras áreas de conhecimento além da computação. Isso se justifica pois jogos sérios no processo de ensino e aprendizagem podem ser desenvolvidos para diferentes contextos e áreas de conhecimento. Para isto seriam necessárias modificações específicas no questionário refletindo o novo contexto.

Outro ponto que contribui para o avanço dessa pesquisa está relacionado ao tratamento de suas limitações. No caso, o modelo MEEGA+, utilizado como base teórica e para construção do instrumento de pesquisa, não foi usado na íntegra, ou seja, na elaboração do questionário foi feita uma abstração das dimensões e elementos dos fatores de qualidade do MEEGA+ a fim de deixar o questionário sucinto e ao mesmo tempo claro para os respondentes. Sendo assim, pode ter havido uma perda na significância dos aspectos abstraídos e consequentemente perda na eficiência da análise dos mesmos. Replicar o questionário utilizando os elementos do MEEGA+ na íntegra e em seguida comparar com os resultados obtidos aqui traria uma melhor compreensão sobre a qualidade dos resultados obtidos neste estudo.

Outra limitação deste trabalho está relacionada aos aspectos de qualidade estudados, já que eles foram baseados apenas nos estudos de Petri e Wangenheim (2019) e de Sales e Silva (2020). Além disso, no presente trabalho são abordados apenas aspectos de qualidade que podem impactar positivamente no jogo, ou seja, aspectos que podem influenciar negativamente no uso de jogos não foram analisados.

\section{Referências}

Barbosa, S. D. J. et al. Interação Humano-Computador e Experiência do Usuário. Autopublicação, 2021.

Battistella, P.E. Games for teaching computing in higher education - a systematic review. IEEE Technology and Engineering Education (ITEE), v.1, n.3, p. 8-30, 32016.

Becker, K. What's the difference between gamification, serious games, educational games, and game-based learning? Academia Letters, Jan 2021. Disponível em: https: //doi.org/10.20935/AL209. 
Brito, R. et al. Macteaching: Utilizando um jogo para apoio ao ensino do método de avaliação de comunicabilidade. Brazilian Symposium on Computers in Education (Simpósio Brasileiro de Informática na Educação - SBIE), v.27, n.1, p.796, 2016.. Disponível em: https://doi.org/10.5753/cbie.sbie.2016.796.

Fullerton, T. Game Design Workshop: A Playcentric Approach to Creating Innovative Games. Taylor and Francis, 2008.

Gerhardt, T. E.; Silveira, D. T. Métodos de pesquisa. 1st. ed. Porto Alegre, Brazil: Editora da UFRGS, 2009.

Kasunic, M. Designing an effective survey, 2005.

NBR-ISO/IEC 9126-1. Rio de Janeiro - RJ, 2003. Engenharia de software - Qualidade de produto Parte 1: Modelo de qualidade.

Nielsen, J. Usability Engineering. Morgan Kaufmann Publishers Inc., 1993.

Petri, G.; Wangenheim, C. G. A method for the evaluation of the quality of games for computing education. In: Anais dos Workshops do VIII Congresso Brasileiro de Informática na Educação (CBIE 2019). Brazilian Computer Society (Sociedade Brasileira de Computação - SBC), 2019. p. 951. Disponível em: https://doi.org/10.5753/ cbie.wcbie.2019.951.

Preece, J.; Rogers, Y.; Sharp, H. Design de Interação. Bookman, 2005.

Queiroz, W.; Beltrão, R.; Fernandes, M.; Bonifácio, B.; Fernandes, P. Macteaching: Uma abordagem para enriquecer o ensino do método de avaliação de comunicabilidade. Anais dos Workshops do Congresso Brasileiro de Informática na Educação, v. 6, n. 1, p. 138, 2017. Disponível: https://doi.org/10.5753/cbie.wcbie.2017.138.

Sales, A. B.; Serrano, M.; Serrano, M. Aprendizagem Baseada em Projetos na Disciplina de Interação Humano-Computador. RISTI - Revista Ibérica de Sistemas e Tecnologias de Informação, scielopt, p.49-64, 2020. Disponível em: https://doi.org/10.17013/risti.37.49-64.

Sales, A. B. de; Antunes, J. G. Evaluation of educational games usage satisfaction. In: 2021 16th Iberian Conference on Information Systems and Technologies (CISTI). 2021. p. 1-6. Disponível em: https://doi.org/10.23919/CISTI52073. 2021.9476400.

Sales, A. B. de; Clímaco, G. de S.; Sales, M. B. de. Jogos sérios em interação humano-computador: Uma revisão sistemática de literatura. RENOTE - Revista Novas Tecnologias na Educação, v.14, n.1, 2016. Disponível em: https: //doi.org/10.22456/1679-1916.67384.

Sales, A. B. de; Costa, E. A. da; Alcantara, P.T.R. de. Evaluation of usability in user satisfaction of electronic commerce. In: 2017 12th Iberian Conference on Information Systems and Technologies (CISTI). 2017. p.1-4. Disponível em: https://doi.org/ 10.23919/CISTI.2017.7975797.

Sales, A. B. de; Silva, M. A. S. e. Jogos sérios no processo de ensino e aprendizagem de interação humano-computador.In: Anais do XXXI Simpósio Brasileiro de Informática na Educação .Porto Alegre, RS, Brasil: SBC, 2020. p. 552-561. Disponível em: https: //doi.org/10.5753/cbie.sbie.2020.552.

SuperData. NY,USA, 2021. 2020 YEAR IN REVIEW.Digital Games and Interactive Media. Disponível em: https://www.digitalmusicnews.com/wp-content/uploads/2021/ 01/SuperData2020YearinReview.pdf.

Vianna, M.; Vianna, Y.; Medina, B.; Tanaka, S.; Krug, M. Gamification, Inc. Como reinventar empresas a partir de jogos. $1^{a}$ edição. ed. : MJV Press, 2013.

Wiemeyer, J.; Nacke, L.; Moser, C.; Mueller,F.F.Player experience. In: .Serious Games. 1st. ed. Springer, 2016. p. 243-271. 